\title{
Detection and Phylogenetic Analysis of Viruses Linked with Fig Mosaic Disease in Seventeen Fig Cultivars in Palestine
}

\author{
Rana Majed Jamous ${ }^{1}$, Salam Yousef Abu Zaitoun', Omar Bassam Mallah ${ }^{1}$, Munqez Shtaya ${ }^{2}$, \\ Toufic Elbeaino ${ }^{3}$, and Mohammed Saleem Ali-Shtayeh ${ }^{1 *}$ \\ ${ }^{1}$ Biodiversity and Environmental Research Center, BERC, Nablus, Palestine \\ ${ }^{2}$ Department of Plant Production and Protection, Faculty of Agriculture and Veterinary Medicine, An-Najah National \\ University, Nablus, Palestine \\ ${ }^{3}$ Istituto Agronomico Mediterraneo di Bari, Via Ceglie 9, 70010 Valenzano, Bari, Italy
}

(Received on January 9, 2020; Revised on April 20, 2020; Accepted on April 21, 2020)

Fig mosaic is a viral disease (FMD) that spreads in Palestinian common fig (Ficus carica L.) orchards. Recognizing the economic value of fig plants and the harmful nature of FMD, the disease poses a significant threat to the economy of the fig production in Palestine. We applied the reverse transcription and amplification (RT-PCR) and PCR technique to leaf samples of 77 trees and 14 seedlings of 17 fig cultivars. The samples were collected from orchards in the main fig-growing provinces of the Palestinian West Bank, to assess the prevalence of viruses associated with FMD, and confirm a possible link of symptoms with viruses detected. Four viruses were detected: Fig mosaic virus (FMV), Fig badnavirus-1 (FBV-1), Fig leaf mottle-associated virus 2 (FLMaV-2), and Fig fleck-associated virus (FFkaV). FMV and FBV-1 were found in all tested fig plants $(100 \%)$, while FLMaV-2 and FFkaV were detected in $61.5 \%$ and $33 \%$ of the fig samples, respectively. The high incidence of FBV-1 in the newly propagated symptomatic and symptomless seedlings from different cultivars may be an indication that FBV-1 is integrated into the genome of the fig in a cultivar nondiscriminatory manner. Very weak or no association was detected between FMD symptoms severity in the 17

\footnotetext{
*Corresponding author

Phone) +970-9-2536406, FAX) +970-9-2536147

E-mail)msshtayeh@yahoo.com

(c) This is an Open Access article distributed under the terms of the Creative Commons Attribution Non-Commercial License (http:// creativecommons.org/licenses/by-nc/4.0) which permits unrestricted noncommercial use, distribution, and reproduction in any medium, provided the original work is properly cited.
}

Articles can be freely viewed online at www.ppjonline.org.
Palestinian fig cultivars with the various viruses' combinations observed (i.e., number of the viruses infecting the plant). These results support the notion that FMD symptom severity expression is likely to be controlled by a combination of FMV infection, cultivars, and environmental factors, rather than the number of viruses infecting the plant.

Keywords : FFkaV, fig, fig viruses, FMD, RT-PCR and sequencing

Handling Editor : Ju-Yeon Yoon

The common fig (Ficus carica L., Moraceae) is one of the earliest cultivated fruit crops in the Mediterranean area that is, in turn, considered the chief fig-producing center worldwide (Ali-Shtayeh et al., 1991; Food and Agriculture Organization of the United Nations, 2009). In general, viral diseases are a major restrictive production factor causing substantial yield losses in crops, and the mosaic disease induced by Fig mosaic emaravirus (FMV; family Fimoviridae, genus Emaravirus) is the most known one on fig. In addition to FMV, numerous viruses have been reported in the tree including six closteroviruses (family Closteroviridae): Fig leaf mottle-associated virus FLMaV-1, FLMaV-2, and FLMaV-3, Fig mild mottle-associated virus FMMaV, and Arkansas fig closterovirus AFCV-1, AFCV-2; badnavirus (Fig badnavirus 1, FBV-1, family Caulimoviridae, genus Badnavirus), macula-like virus (Fig fleck-associated virus, FFkaV, family Tymoviridae, genus Maculavirus); deltapartitivirus (Fig cryptic virus 1, FCV-1, family Partitiviridae, genus Alphacryptovirus); trichovirus (Fig latent virus 1, FLV-1, family Betaflexiviridae genus 
Table 1. Prevalence of some fig viruses in different fig-growing areas in the world

\begin{tabular}{|c|c|c|c|c|c|c|c|c|c|}
\hline \multirow{2}{*}{ Area } & \multicolumn{8}{|c|}{ Fig viruses ${ }^{\mathrm{a}}$} & \multirow{2}{*}{ Reference } \\
\hline & FMV & FFkaV & FLMaV-1 & FLMaV-2 & FMMaV & FLV-1 & FCV & FBV-1 & \\
\hline Turkey & $+\left(1^{\mathrm{st}}\right)^{\mathrm{b}}$ & NT & - & $+\left(2^{\text {nd }}\right)^{b}$ & NT & NT & NT & NT & Caglar et al. (2011) \\
\hline Egypt & $+\left(2^{\text {nd }}\right)^{b}$ & NT & $+\left(1^{\mathrm{st}}\right)^{\mathrm{b}}$ & + & + & NT & NT & NT & Elbeshehy and Elbeaino (2011) \\
\hline Lebanon & $+\left(2^{\text {nd }}\right)^{b}$ & + & $+\left(1^{\mathrm{st}}\right)^{\mathrm{b}}$ & + & + & NT & NT & NT & Elbeaino et al. (2012c) \\
\hline Iran & + & NT & $+\left(2^{\text {nd }}\right)^{b}$ & - & - & $+\left(1^{\mathrm{st}}\right)^{\mathrm{b}}$ & NT & NT & Shahmirzaie et al. (2012) \\
\hline Syria & $+\left(1^{\mathrm{st}}\right)^{\mathrm{b}}$ & $+\left(2^{\text {nd }}\right)^{b}$ & + & + & + & + & - & NT & Elbeaino et al. (2012a) \\
\hline Tunisia & $+\left(1^{\mathrm{st}}\right)^{\mathrm{b}}$ & + & + & + & + & $+\left(2^{\text {nd }}\right)^{\mathrm{b}}$ & + & NT & El Air et al. (2015) \\
\hline Saudi Arabia & $+\left(2^{\text {nd }}\right)^{b}$ & NT & $+\left(1^{\mathrm{st}}\right)^{\mathrm{b}}$ & + & + & NT & NT & NT & Aldhebiani et al. (2015) \\
\hline China & $+\left(2^{\text {nd }}\right)^{b}$ & + & + & - & + & - & - & $+\left(1^{\mathrm{st}}\right)^{\mathrm{b}}$ & Mijit et al. (2017) \\
\hline $\begin{array}{l}\text { Bosnia \& Herzegovina, } \\
\text { and Montenegro }\end{array}$ & + & + & $+\left(2^{\text {nd }}\right)^{b}$ & + & + & - & - & $+\left(1^{\mathrm{st}}\right)^{\mathrm{b}}$ & Delić et al. (2017) \\
\hline Montenegro & + & - & $+\left(2^{\text {nd }}\right)^{\mathrm{b}}$ & - & + & - & - & $+\left(1^{\mathrm{st}}\right)^{\mathrm{b}}$ & Latinović et al. (2019) \\
\hline
\end{tabular}

${ }^{a}$ Fig viruses: FMV, Fig mosaic virus; FFkaV, Fig fleck-associated virus; FLMaV-1, Fig leaf mottle-associated virus 1; FLMaV-2, Fig leaf mottle-associated virus 2; FMMaV, Fig mild mottle-associated virus; FLV-1, Fig latent virus 1; FCV, Fig cryptic virus; FBV-1, Fig badnavirus-1.

${ }^{\mathrm{b}}$ The most prevalent viruses; +, virus detected; -, virus not detected; NT, virus not tested.

Trichovirus); and waikavirus (Strawberry latent ringspot virus, SLRSV, family Comoviridae, genus Nepovirus) and three viroids (Hop stunt viroid [HSVd], Citrus exocortis viroid [CEVd], and Apple dimple fruit viroid [ADFVd]) (Elbeaino et al., 2006, 2007, 2009b, 2011a, 2012b, 2014; Minafra et al., 2012; Norozian et al., 2014; Tzanetakis et al., 2010). Undoubtedly, the fig mosaic disease (FMD) is the most widespread viral disease of fig, which represents a threat and a continuous constraint for healthy fig production and germplasm exchange worldwide (Shahmirzaie et al., 2012).

Different viruses have been found in several fig-growing areas worldwide (Table 1). In Syria and Tunisia, six and seven viruses were detected in fig trees, respectively, with FMV the predominant virus (El Air et al., 2015; Elbeaino et al., 2012a). Also, in Tunisia, FLV-1 was observed in all studied areas in symptomatic as well as symptomless trees. In Iran, Shahmirzaie et al. (2012) detected three viruses: FLV-1 (predominant), FLMaV-1, and FMV in fig trees. In Lebanon, Egypt, and western Saudi Arabia, 4-5 viruses were observed in fig trees with FLMaV-1 as the predominant, followed by FMV (Aldhebiani et al., 2015; Elbeaino et al., 2012c; Elbeshehy and Elbeaino, 2011). Following the discovery of FBV-1, the only DNA virus described in fig to date, in an FMD tree in the United States in 2008 (Tzanetakis et al., 2010), the virus was found to be one of the most widespread viruses in various fig-growing areas of the world (Delić et al., 2017; Laney et al., 2012; Latinović et al., 2019; Mijit et al., 2017).

FMV belongs to the genus Emaravirus, is transmitted by the eriophyid mite Aceria ficus Cotte (Flock and Wallace, 1955). On the other hand, several closteroviruses (e.g., FLMaV-2), are presumably transmitted by aphids (Nahdi et al., 2006). Badnaviruses are spread mainly by mealybugs and aphids, and thus FBV-1 may share vectors with the closteroviruses that infect the fig tree (Tzanetakis et al., 2010).

Excluding FMV, not much information is known about the etiology of other viruses in symptoms induction. However, symptoms similar to mosaic disease have been recurrently observed in FMV-free fig plants infected with a mixture of fig viruses (Elbeaino et al., 2006, 2007, 2010, 2011a, 2011b). No fig virus is transmitted through seeds, contrarily to FBV-1, which is vertically transmitted to seedlings without inducing symptoms (Minafra et al., 2012). The lack of complete association between FMV and FMD, detection of numerous new viruses in fig mosaic trees, inability to complete Koch postulates, and extreme symptom variability, indicate that the disease is more complicated than originally assumed and symptoms may not be only instigated by FMV but also by mixed virus infections (Laney et al., 2012; Susaimuthu et al., 2008).

In Palestine, FMD was initially noticed in 1955 (Goor, 1955). Later, the disease has been observed on figs yearly, 
with symptoms varying depending on the season, variety, and level of fig mite (Aceria ficus) infestation (Ali-Shtayeh et al., 1991). However, very little information is available on the viruses associated with FMD in Palestine. In 2015, Alkowni et al. (2015) analyzed a few fig leaf samples (25) of unknown cultivars from three nearby villages in Nablus District, in addition to six newly propagated seedlings of unverified cultivars obtained from a local nursery. The study reported the presence of four viruses: FMV, FBV1, FLV-1, and FLMaV-2, with FBV-1 followed by FMV, were the most common viruses detected in the studied delimited area.

Fig landraces are expected to represent a source of beneficial genes with a high capacity to tolerate living and nonliving pressures, resulting in excessive produce constancy, disease resistance, and reasonable produce level under low external agricultural input (Ali-Shtayeh et al., 2014; Zeven, 1998). However, information regarding the presence and incidence of viral infections in fig cultivars in Palestine is still scarce.

This present study aimed to explore the incidence of viral infection (eight viruses) in 17 fig cultivars growing in the main fig-producing areas in Palestine, and to study the se- quences of the observed viruses in comparison with homologs reported in the GenBank database, establish a possible association between symptoms and the viruses present in different Palestinian fig cultivars, and attempt to identify genotypes that could serve as sources of resistance to virus infection.

\section{Materials and Methods}

Leaf sample collection. FMD like symptoms were detected in 2016 on diverse local fig cultivars in 24 orchards in the main fig-producing areas of the West Bank in Palestine (Fig. 1). The surveying and collection of samples were carried out in May 2017. During surveying, leaf samples were collected from 77 trees of the main 17 cultivated fig cultivars in Palestine. In 2019, leaf samples were also collected from 14, 2-year-old fig seedlings of ten different cultivars propagated by hardwood cuttings from fig orchards in the main fig-producing areas of Palestine.

FMD symptoms and severity. The incidence and severity percentage of FMD symptoms in fig cultivars were inspected, and symptoms were documented and photo-

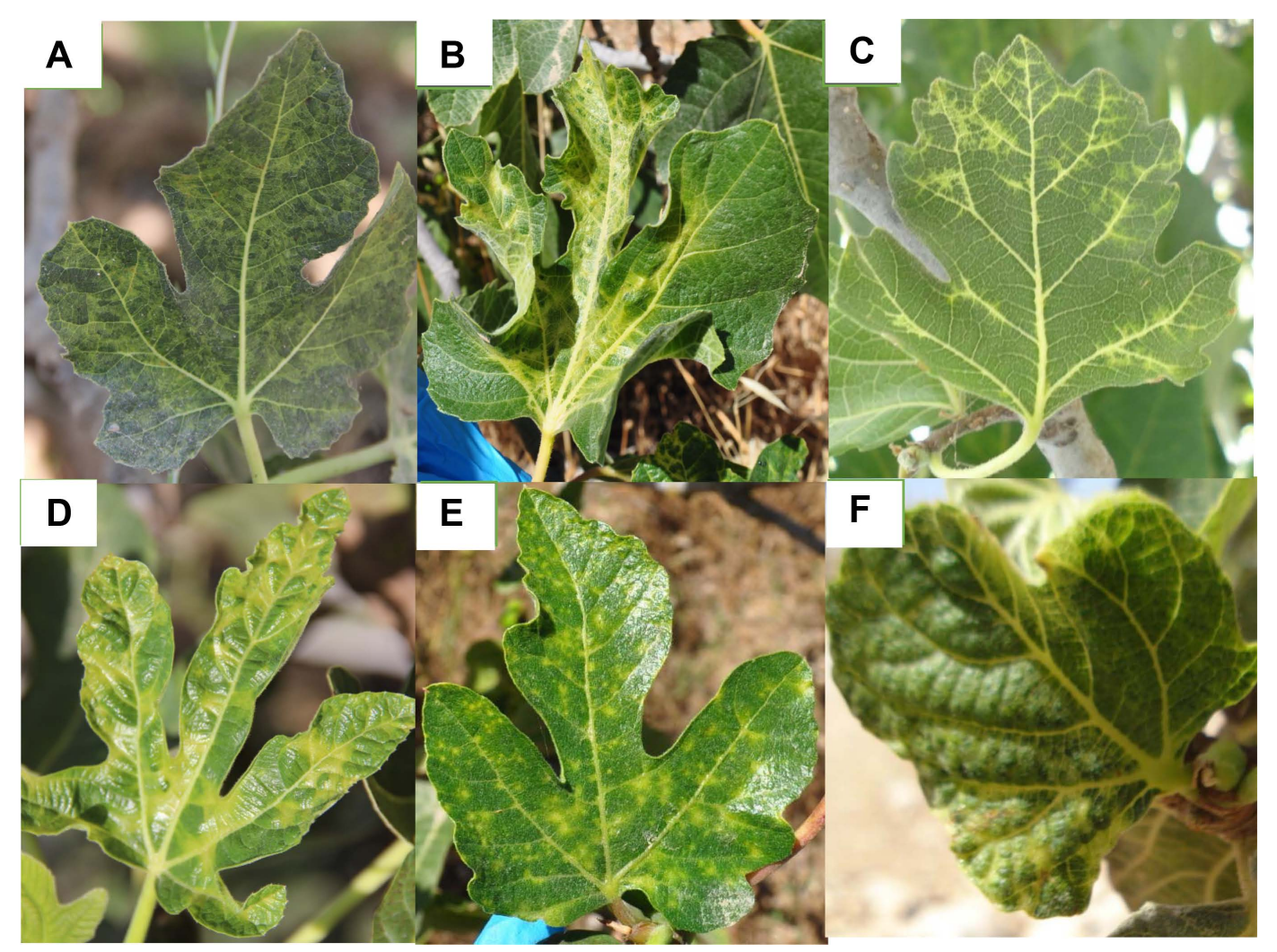

Fig. 1. Different types of symptoms of the mosaic disease on leaves of inspected fig trees in the collection plot: leaf discoloration (A), deformation and chlorotic mottling (B), vein banding (C), chlorosis (D), chlorotic ring spots (E), and leaf puckering (F). 
graphed (Fig. 1). Twenty fully developed leaves, from each fig cultivar, were randomly collected to evaluate symptom severity, according to the percentage of the leaf area with symptoms (\%LAWS). The collected leaves displayed signs expected to be of viral source and not to have been affected by any other reason. The upper leaf surface was scanned using the Epson scanner (Epson L355, Pasig, Philippines) and saved in a jpg format. The resulted images were exposed to the Leaf Doctor integrative smartphone application, which was used to distinguish between diseased and healthy plant tissues and estimate the \%LAWS representing disease severity as described by Pethybridge and Nelson (2015).

The presence or absence of FMD symptoms was scored for each collected leaf sample. Disease incidence was calculated as the percentage of leaves with specific signs out of those surveyed.

Extraction of total nucleic acids (TNAs). Leaf veins tissues from symptomless samples and leaf samples showing mosaic-like symptoms were used to extract TNAs using a silica-capture-based extraction method, according to Foissac et al. (2001), with minor modifications. Briefly, a total of $100 \mathrm{mg}$ of tissue was ground under liquid nitrogen and mixed with $1 \mathrm{ml}$ buffer solution (4.0 M guanidine thiocyanate, $0.2 \mathrm{M} \mathrm{NaOAc}$ [pH 5.2], 25 mM EDTA, 1.0 M KOAc and $2.5 \% \mathrm{w} / \mathrm{v}$ PVP-40). The supernatant was transferred to a new tube containing $100 \mu \mathrm{l}$ of $10 \% \mathrm{~N}$ lauroyl sarkosyl and incubated at $70^{\circ} \mathrm{C}$ for 10 min with intermittent shaking, followed by incubation in ice for $5 \mathrm{~min}$, and centrifuge for $10 \mathrm{~min}$. Five hundred microliters of the supernatant was transferred to a new tube containing $150 \mu \mathrm{l}$ of ethanol, $100 \mu 1$ of suspended silica (Sigma-Aldrich, Darmstadt, Germany) and $300 \mu$ of $6 \mathrm{M}$ sodium iodide. The obtained silica pellet was washed twice in washing buffer $(10 \mathrm{mM}$ Tris $\mathrm{pH}$ 7.5, $0.5 \mathrm{mM}$ EDTA, $50 \mathrm{mM} \mathrm{NaCl}, 50 \%$ ethanol), briefly dried and nucleic acids were eluted by suspension in $200 \mu \mathrm{l}$ of nuclease-free water, the silica was then collected by centrifugation for $3 \mathrm{~min}$, and $150 \mu \mathrm{l}$ of the sample was transferred to a clean tube. The yield and quality of TNAs were measured using the Epoch Microplate spectrophotometer (Gene5 Take 3 Module, Winooski, VT, USA), and samples were stored at $-80^{\circ} \mathrm{C}$ until used.

Reverse transcription polymerase chain reaction (RTPCR) assays. For cDNA synthesis from the target RNA, a two-step reverse transcription and amplification (RT-PCR) protocol was used. Briefly, $10 \mu \mathrm{l}$ of TNA extracts were mixed with $0.5 \mu \mathrm{g}$ of random hexamer primers (Roche Diagnostic, Munich, Germany). The mixture was denatured for $5 \mathrm{~min}$ at $95^{\circ} \mathrm{C}$ and quickly chilled on ice. RT-PCR was performed for $1 \mathrm{~h}$ at $39^{\circ} \mathrm{C}$ by adding $2 \mu \mathrm{l}$ of $10 \times$ Moloney

Table 2. List of primers used in RT-PCR assays for the detection of fig viruses

\begin{tabular}{|c|c|c|c|c|}
\hline Viruse & Primer name & Primer sequence & Amplicons (bp) & Reference \\
\hline \multirow[t]{2}{*}{ FMV } & E5s & 5'-CGGTAGCAAATGGAATGAAA-3' & 302 & Elbeaino et al. (2009a) \\
\hline & E5a & 5'-AACACTGTTTTTGCGATTGG-3' & & \\
\hline \multirow[t]{2}{*}{ FBV-1 } & P1s & 5'-GCTGATCACAAGAGGCATGA-3' & 214 & Tzanetakis et al. (2010) \\
\hline & P1a & 5'-TCCTTGTTTCCACGTTCCTT-3' & & \\
\hline \multirow[t]{2}{*}{ FLMaV-2 } & $\mathrm{F} 3 \mathrm{~s}$ & 5'-GAACAGTGCCTATCAGTTTGATTTG-3' & 360 & Elbeaino et al. (2007) \\
\hline & $\mathrm{F} 3 \mathrm{a}$ & 5'-TCCCACCTCCTGCGAAGCTAGAGAA-3' & & \\
\hline \multirow[t]{2}{*}{ FFkaV } & D8s & 5'-ATGACGACTGTCAACTCCCT-3' & 270 & Elbeaino et al. (2011a) \\
\hline & D8a & 5'-TTAAGCCAGGGTGGGAGTGTTG-3' & & \\
\hline \multirow[t]{2}{*}{ FLMaV-1 } & $\mathrm{N} 17 \mathrm{~s}$ & 5'-CGTGGCTGATGCAAAGTTTA-3' & 350 & Elbeaino et al. (2006) \\
\hline & $\mathrm{N} 17 \mathrm{a}$ & 5'-GTTAACGCATGCTTCCATGA-3' & & \\
\hline \multirow[t]{2}{*}{ FMMaV } & LM3s & 5'-AAGGGGAATCTACAAGGGTCG-3' & 311 & Elbeaino et al. (2010) \\
\hline & LM3a & 5'-TATTACGCGCTTGAGGATTGC-3' & & \\
\hline \multirow[t]{2}{*}{ FCV-1 } & R1s & 5'-TCGATTGTCTTTGGAGAGG-3' & 353 & Elbeaino et al. (2011a) \\
\hline & R1a & 5'-CGCATCCACAGTATCCCATT-3' & & \\
\hline \multirow[t]{2}{*}{ FLV-1 } & CPtrs & 5'-CСАТСTTCACCACACAAATGTC-3' & 389 & Gattoni et al. (2009) \\
\hline & CPtra & 5'-CAATCTTCTTGGCCTCCATAAG-3' & & \\
\hline
\end{tabular}

RT-PCR, reverse transcription and amplification; FMV, Fig mosaic virus; FBV-1, Fig badnavirus-1; FLMaV-2, Fig leaf mottle-associated virus 2; FFkaV, Fig fleck-associated virus; FLMaV-1, Fig leaf mottle-associated virus 1; FMMaV, Fig mild mottle-associated virus; FCV-1, Fig cryptic virus 1; FLV-1, Fig latent virus 1. 
Murine Leukaemia Virus (M-MLV) buffer, $2 \mu 1$ of $10 \mathrm{mM}$ dithiothreitol, $0.5 \mu 1$ of $10 \mathrm{mM}$ dNTPs, and 200 units of M-MLV reverse transcriptase enzyme (Thermo Fisher, Waltham, MA, USA) in a final volume of $20 \mu \mathrm{l}$.

The detection of FLMaV-1, FLMaV-2, FMMaV, FMV, FFkaV, FCV-1, and FLV-1 was conducted with RT-PCR, whereas FBV-1 with PCR, using eight sets of specific primers selected based on previously published papers (Table 2). Briefly, $2.5 \mu \mathrm{l}$ of the cDNA product was used for amplification with the addition of $12.5 \mu \mathrm{l}$ of GoTaq Green Master Mix (Promega, Madison, WI, USA), with a final volume of $25 \mu \mathrm{l}$. cDNAs were amplified in the thermal cycler GeneAmp PCR System 9700 (Applied Biosystems, Beverly, MA, USA) with 35 cycles at $92^{\circ} \mathrm{C}$ for $30 \mathrm{~s}, 58^{\circ} \mathrm{C}$ for $45 \mathrm{~s}\left(50^{\circ} \mathrm{C}\right.$ for $\left.\mathrm{FBV}-1\right), 72^{\circ} \mathrm{C}$ for $1 \mathrm{~min}$. In the last cycle, extension at $72^{\circ} \mathrm{C}$ for 5 min was performed. The
RT-PCR products were analyzed on $1.2 \%$ agarose gels in $1 \times$ TAE buffer (Sambrook et al., 1989), and visualized by UV illumination using the GelRed and Gel Documentation System (Syngene, Cambridge, UK).

PCR amplicons were sequenced bi-directionally using forward and reverse virus-specific primers (Table 2) (Macrogen, Seoul, Korea). Nucleotide sequences were analyzed with the assistance of the Chromas program (Technelysium Pty Ltd., Brisbane, Australia). Different sequences of related viruses infecting figs (see Supplementary Table 1) were retrieved from the GenBank (http://www. ncbi.nlm.nih.gov) and used for comparison using CLC Main Workbench 8.0.1 software (CLC bio A/S, Aarhu, Denmark). After multiple sequence alignments, phylogenetic trees have been constructed using the UPGMA algorithm of the CLC Main Workbench 8.0.1 software, with

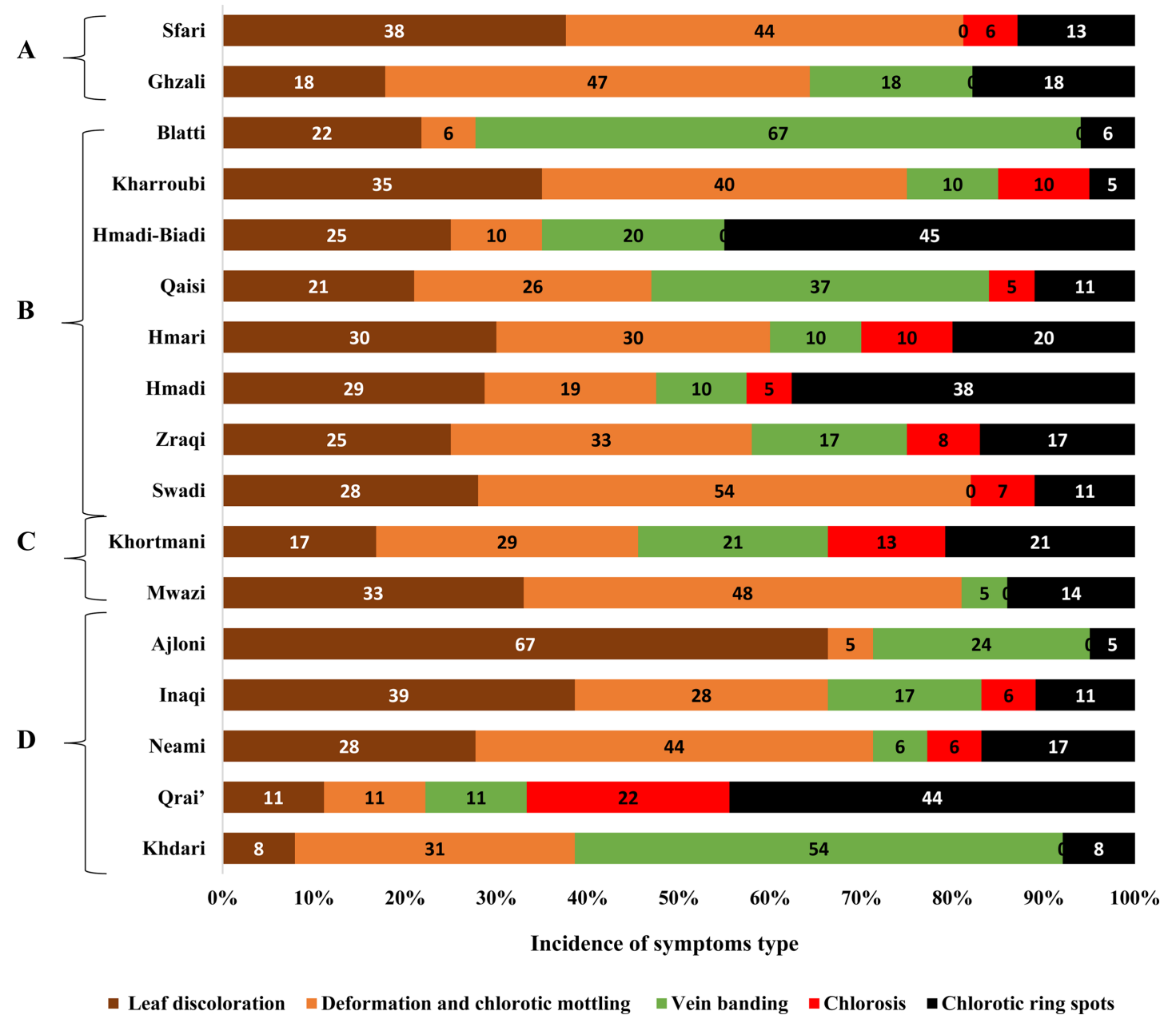

Fig. 2. Incidence of different symptom types in fig mosaic infected trees in 17 Palestinian fig cultivars. A, varieties infected with Fig mosaic virus (FMV) and Fig badnavirus-1 (FBV-1); B, varieties infected with FMV, FBV-1, and Fig leaf mottle-associated virus 2 (FLMaV-2); C, varieties infected with FMV, FBV-1, and Fig fleck-associated virus (FFkaV); D, varieties affected with FMV, FBV-1, FFkaV, and FLMaV-2. 

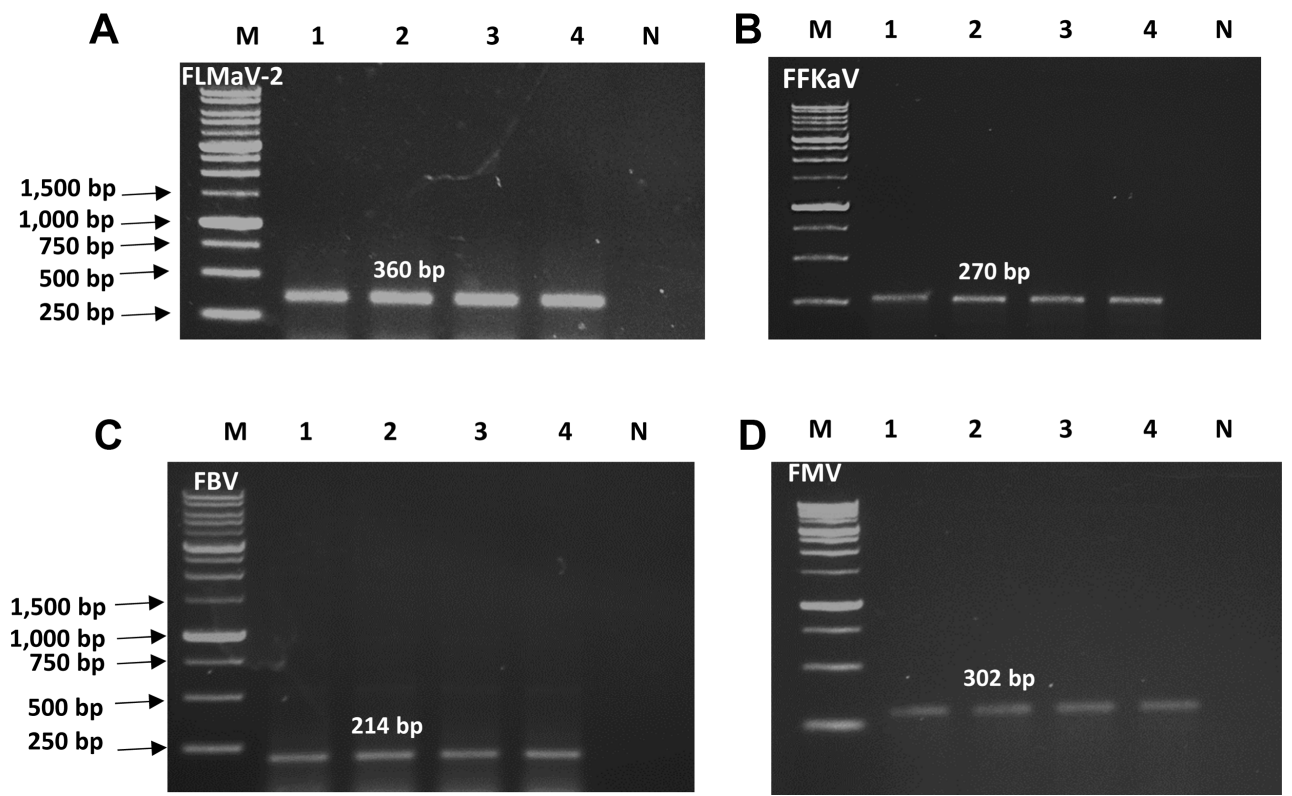

Fig. 3. Electrophoretic profile of reverse transcription and amplification products of infected figs with Fig leaf mottle-associated virus-2 (FLMaV-2) (A), Fig fleck-associated virus (FFkaV) (B), Fig badnavirus-1 (FBV-1) (C), and Fig mosaic emaravirus (FMV) (D). Lane M, DNA ladder marker; lanes 1, 2, 3, 4, and 5, PCR-positive infected fig plants; N, negative control.

1,000 bootstrap replicates.

\section{Results}

Mosaic disease symptoms in fig. The visual disease monitoring for FMD in May 2017 revealed around 100\% disease incidence in all examined cultivars. Various symptoms noted in all different cultivars, including ring spots, chlorotic mottling, blotching, mosaic, and vein banding (Fig. 1).

The most abundant FMD symptoms on the leaves of the different cultivars were: deformation and chlorotic mottling, with symptom incidence range of $29-54 \%$ in Swadi, Neami, Mwazi, Khortmani, Zraqi, Ghzali, and Sfari; welldefined rings (38-45\%) in Qra'i, Hmadi, and Hmadi-Biadi; vein banding (37-67\%) in Khdari, Qaisi, and Blati; and leaf discoloration (35-67\%) in Inaqi, Ajloni, and Kharoubi cultivars (Fig. 2).

RT-PCR detection of fig viruses in the Palestinian fig cultivars. The specific primers used in different RT-PCR and PCR assays were efficient to detect fig viruses in all samples collected from the 17 common fig cultivars in Palestine, thus generating amplicons of the expected size for FLMaV-2, FFkaV, FBV-1, and FMV (Fig. 3); whereas FLMaV-1, FMMaV, FCV-1, and FLV-1 were not detected.

The results show that all surveyed FMD trees of the different cultivars were infected with at least two viruses
(FMV, and FBV-1), and 67.5\% (52/77) of these trees contained three viruses or more. Noteworthy was the high incidence of FMV and FBV-1 in all trees (100\% of infection), whereas FLMaV-2 and FFkaV were present to a lesser extent, with $54.5 \%$ (42/77) and $20.8 \%$ (16/77) of infection rate, respectively (Table 3 ).

However, the incidence of FLMaV-2 and FFkaV varied, according to cultivar (Table 3). FLMaV-2 was detected in $76.47 \%(13 / 17)$ of the surveyed cultivars. It was most prevalent in the Inaqi, Qaisi, Blatti, and Swadi (100\%), followed by Hmari and Qra'i (80\%), and the remaining FLMaV-2-infected cultivars (25-60\%). However, it was not detected in the Khortmani, Sfari, Mwazi, and Ghzali. On the other hand, FFkaV was detected in $41.17 \%(7 / 17)$ of the surveyed cultivars and was most prevalent in the Mwazi (100\%), followed by Ajloni (75\%), and the remaining FFkaV-infected cultivars (25-60\%) (Table 3). Furthermore, the FLMaV-2 and FFkaV incidence was varied in a consistent way with the geographical locations (Fig. 4). The FLMaV-2 infection rates in the West Bank of Palestine in decreasing order were $65 \%$ in the Southern West Bank, 53.8\% in Central West Bank, and 48\% in Northern West Bank. On the other hand, FFkaV infection rates were the highest in Northern West Bank (35.5\%), followed by Central West Bank (11.5\%), and Southern West Bank (11\%) (Table 3, Fig. 4).

All of the studied cultivars were infected with at least two viruses (FMV and FBV-1). The incidence rate for all 
Table 3. Incidence of fig viruses detected in leaves of the sampled trees of different fig cultivars in fig-growing areas in Palestine

\begin{tabular}{|c|c|c|c|c|c|c|}
\hline Variety & Fruit skin color & Province & FMV & FBV-1 & FLMaV-2 & FFkaV \\
\hline Inaqi & Green-Purple & NWB & $5 / 5(100)$ & $5 / 5(100)$ & $5 / 5(100)$ & $3 / 5(60)$ \\
\hline Khortmani & Green-Purple & & $4 / 4(100)$ & $4 / 4(100)$ & $0 / 4(0)$ & $1 / 4(25)$ \\
\hline Qra'i & Green-yellow & & $5 / 5(100)$ & $5 / 5(100)$ & $4 / 5(80)$ & $2 / 5(40)$ \\
\hline Mwazi & Green-Yellow & & $4 / 4(100)$ & $4 / 4(100)$ & $0 / 4(0)$ & $4 / 4(100)$ \\
\hline Hmadi & Green-Purple & & $5 / 5(100)$ & $5 / 5(100)$ & $3 / 5(60)$ & $0 / 5(0)$ \\
\hline Zraqi & Black-Violet & & $4 / 4(100)$ & $4 / 4(100)$ & $1 / 4(25)$ & $0 / 4(0)$ \\
\hline Khdari & Green-Yellow & & 4/4 (100) & 4/4 (100) & $2 / 4(50)$ & $1 / 4(25)$ \\
\hline Sub-Total & & & $31 / 31(100)$ & $31 / 31(100)$ & $15 / 31(48.3)$ & $11 / 31(35.5)$ \\
\hline Ghzali & Bronze with purple ribs & CWB & $4 / 4(100)$ & 4/4 (100) & $0 / 4(0)$ & $0 / 4(0)$ \\
\hline Kharroubi & Black-Violet & & $5 / 5(100)$ & $5 / 5(100)$ & $2 / 5(40)$ & $0 / 5(0)$ \\
\hline Qaisi & Black-Violet & & 4/4 (100) & $4 / 4(100)$ & 4/4 (100) & $0 / 4(0)$ \\
\hline Ajloni & Green-Yellow & & $4 / 4(100)$ & $4 / 4(100)$ & $2 / 4(50)$ & $3 / 4(75)$ \\
\hline Blatti & Green-Yellow & & 4/4 (100) & 4/4 (100) & 4/4 (100) & $0 / 4(0)$ \\
\hline Hmadi-Biadi & Green-Yellow & & $5 / 5(100)$ & $5 / 5(100)$ & $2 / 5(40)$ & $0 / 5(0)$ \\
\hline Sub-Total & & & $26 / 26(100)$ & $26 / 26(100)$ & $14 / 26(53.8)$ & $3 / 26(11.5)$ \\
\hline Hmari & Green-Purple & SWB & $5 / 5(100)$ & $5 / 5(100)$ & $4 / 5(80)$ & $0 / 5(0)$ \\
\hline Neami & Green-Yellow & & $5 / 5(100)$ & $5 / 5(100)$ & $3 / 5(60)$ & $2 / 5(40)$ \\
\hline Sfari & Green-Yellow & & 4/4 (100) & 4/4 (100) & $0 / 4(0)$ & $0 / 4(0)$ \\
\hline \multirow[t]{2}{*}{ Swadi } & Black-Purple & & 6/6 (100) & 6/6 (100) & $6 / 6(100)$ & $0 / 6(0)$ \\
\hline & Sub-Total & & $20 / 20(100)$ & $20 / 20(100)$ & $13 / 20(65)$ & $2 / 20(11)$ \\
\hline Total & & & 77/77 (100) & 77/77 (100) & $42 / 77(54.5)$ & $16 / 77(22.8)$ \\
\hline
\end{tabular}

Values are presented as number (\%).

FMV, Fig mosaic virus; FBV-1, Fig badnavirus-1; FLMaV-2, Fig leaf mottle-associated virus 2; FFkaV, Fig fleck-associated virus; NWB, Northern West Bank (Jenin, Tulkarm, Qalqiliah, Tubas, Salfit, Nablus); CWB, Central West Bank (Ramallah, Jerusalem, Jericho); SWB, Southern West Bank (Hebron, Bethlehem).

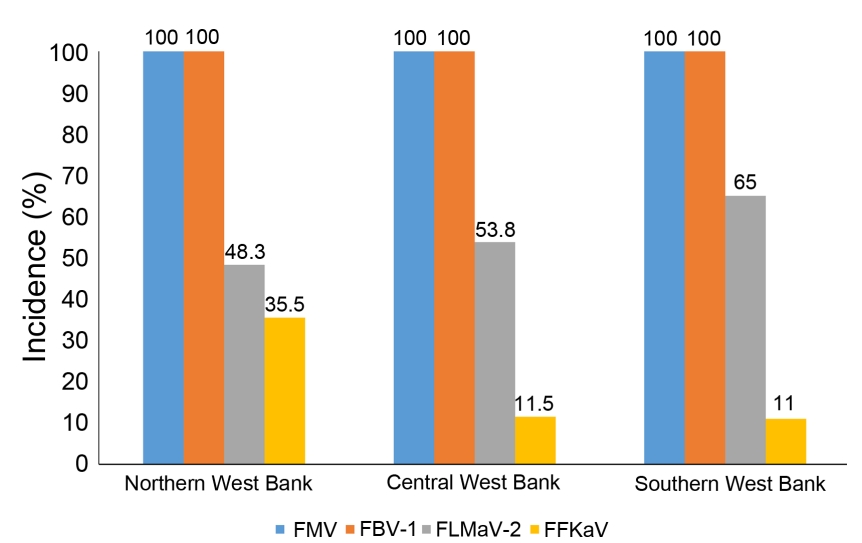

Fig. 4. Incidence of fig viruses' infections in fig samples collected from orchards in northern, central, and southern provinces of the Palestinian West Bank. FMV, Fig mosaic virus; FBV-1, Fig badnavirus-1; FLMaV-2, Fig leaf mottle-associated virus 2; FFkaV, Fig fleck-associated virus. detected viruses in all surveyed trees in each cultivar, out of the total signals, was expressed as the percentage of positive signals. The percentage of positive signals was calculated by dividing the number of positive RT-PCR for all trees in each cultivar by the total number of trees of the same cultivar multiplied by the number of viruses ( 4 viruses). Mixed infections, in addition to FMV and FBV1, were as follows: five cultivars (29.4\%) had FLMaV-2 and FFkaV, eight (47.1\%) had FLMaV-2, and two (11.8\%) had FFkaV; the remaining two (11.8\%) had only FMV and FBV-1 (Table 4).

The highest percentage of positive RT-PCR signals for all surveyed cultivars were detected in Inaqi, Ajloni, and Qra'i 90.0, 81.3, and 80.0\%, respectively. On the other hand, Hmadi, Hmadi-Biadi, Kharroubi, Khortmani, Zraqi, Ghzali, and Sfari show lower percentages of positive signals with a range of (50.0-65.0\%) (Table 4).

The results also show that all surveyed symptomatic and 
Table 4. The relationship between the percentage of positive signals of fig-infecting viruses combinations and $\%$ of leaf area with fig mosaic disease symptoms (\%LAWS)

\begin{tabular}{lcccc}
\hline Fig variety & $\begin{array}{c}\text { \% of positive } \\
\text { signals }\end{array}$ & $\begin{array}{c}\text { Possible symptoms- } \\
\text { inducing viruses }\end{array}$ & \%LAWS & $\begin{array}{c}\text { Range of LAWS values } \\
\text { per individual leaf (\%) }\end{array}$ \\
\hline Inaqi & 90.0 & FMV, FBV-1, FLMaV-2, FFkaV & 12 & $0.6-41.6$ \\
Ajloni & 81.3 & & 13 & $0.6-42.1$ \\
Qra'i & 80.0 & & 6 & $0.4-20.3$ \\
Neami & 75.0 & & 9 & $0.03-27.9$ \\
Khdari & 68.8 & & 8 & $2.8-18.8$ \\
Mwazi & 75.0 & FMV, FBV-1, FFkaV & 13 & $1.9-23.4$ \\
Khortmani & 56.3 & & $16^{\mathrm{a}}$ & $2.7-28.7$ \\
Swadi & 75.0 & FMV, FBV-1, FLMaV-2 & $14^{\mathrm{a}}$ & $1.7-27.5$ \\
Qaisi & 75.0 & & 10 & $1.0-23.7$ \\
Blatti & 75.0 & & $13^{\mathrm{a}}$ & $0.7-32.7$ \\
Hmari & 70.0 & & 9 & $2.8-34.7$ \\
Hmadi-Biadi & 60.0 & & 12 & $2.3-27.3$ \\
Kharroubi & 60.0 & & 12 & $1.7-26.7$ \\
Zraqi & 56.3 & & 2 & $0.04-5.5$ \\
Hmadi & 65.0 & & 7 & $0.14-18.3$ \\
Ghzali & 50.0 & FMV, FBV-11 & 8 & $2.0-20.9$ \\
Sfari & 50.0 & & $14^{\mathrm{a}}$ & $4.1-25.4$ \\
\hline
\end{tabular}

FMV, Fig mosaic virus; FBV-1, Fig badnavirus-1; FLMaV-1, Fig leaf mottle-associated virus 1; FFkaV, Fig fleck-associated virus; FLMaV-2, Fig leaf mottle-associated virus 2.

${ }^{a}$ Cultivars with the most severe symptom expression.

Table 5. Incidence of fig viruses detected in leaves of the sampled seedlings produced by clonal propagation of hardwood cuttings collected from fig trees of different fig varieties in figgrowing areas in Palestine

\begin{tabular}{|c|c|c|c|}
\hline \multirow[b]{2}{*}{ Fig variety } & \multirow[b]{2}{*}{$\begin{array}{l}\text { No. of seed- } \\
\text { lings/ } \\
\text { cultivar }\end{array}$} & \multicolumn{2}{|c|}{ Viruses incidence } \\
\hline & & $\begin{array}{l}\text { FMV, FBV-1, } \\
\text { FLMaV-2 }\end{array}$ & $\begin{array}{c}\text { FLMaV, FFkaV, } \\
\text { FCV-1, FM- } \\
\text { MaV, FLV-1 }\end{array}$ \\
\hline Ajloni & 1 & $1 / 1(100)$ & 0 \\
\hline Blatti & 2 & $2 / 2(100)$ & 0 \\
\hline Hmadi & 1 & 1/1 (100) & 0 \\
\hline Hmadi-Biadi & 1 & 1/1 (100) & 0 \\
\hline Hmari & 2 & $2 / 2(100)$ & 0 \\
\hline Inaqi & 1 & 1/1 (100) & 0 \\
\hline Khdari & 1 & 1/1 (100) & 0 \\
\hline Neami & 2 & $2 / 2(100)$ & 0 \\
\hline Qaisi & 1 & 1/1 (100) & 0 \\
\hline Qra'i & 2 & $2 / 2(100)$ & 0 \\
\hline
\end{tabular}

FMV, Fig mosaic virus; FBV-1, Fig badnavirus-1; FLMaV-2, Fig leaf mottle-associated virus 2; FFkaV, Fig fleck-associated virus; FCV-1, Fig cryptic virus 1; FMMaV, Fig mild mottle-associated virus; FLV-1, Fig latent virus 1. asymptomatic fig seedlings of ten different cultivars have shown a $100 \%$ infection rate with three viruses (FMV, FBV-1, and FLMaV-2) (Table 5).

Processing of images with leaf symptoms and estimation of FMD severity. Data obtained from processing the scanned images with FMD symptoms using the Leaf Doctor application provided differences of symptom severity concerning different fig cultivars (Table 4). Consequently, the calculated data showed that Zraqi had the lowest \%LAWS (2\%), i.e., the least disease severity. Varieties with moderate expression of symptoms were Qra'i, Hmadi, Ghzali, Khdari, Hmari, Neami, and Qaisi (6-10\%). HmadiBiadi, Kharroubi, Inaqi, Mwazi, Blati, Ajloni, Sfari, Swadi, Khortmani were the cultivars with the most severe symptom expression (12-16\%). However, a very weak correlation $\left(r^{2}=0.05\right)$ was detected between disease severity in each cultivar, in terms of \%LAWS, and the percentage of positive RT-PCR signals.

Phylogenetic analyses of fig-infecting viruses detected in this study. At the sequence level, eight viral DNA has been obtained from sequencing RT-PCR and PCR ampli- 
cons generated from different fig trees infected with FMV, FLMaV-2, FFkaV, and FBV-1. The sequences were deposited in the GenBank under accession numbers MN603621 and MN603622 (FMV); MN603623 and MN603624 (FLMaV-2); MN603625 and MN603626 (FFkaV); MN603619 and MN603620 (FBV-1). These were used to determine the genetic variability of the isolated viruses from Palestine in comparison with previous reports from different areas.

The nucleotide analyses of the four recorded viruses in this study (FMV, FBV-1, FFkaV, and FLMaV-2) showed that the two sequences of FBV-1 and FMV amplicons
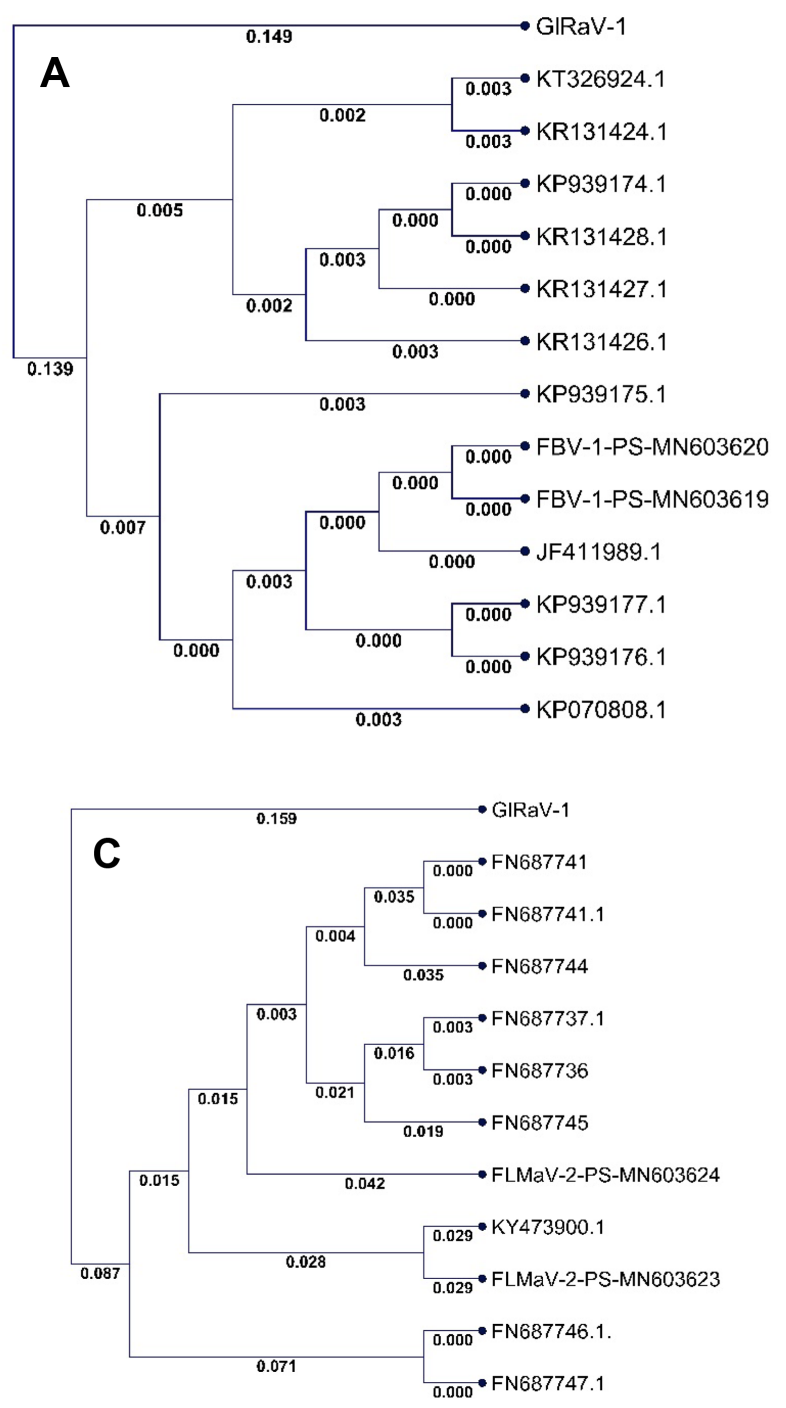

generated from different infected plants had $100 \%$ identity between the two isolates for each virus, however, these isolates showed $97.3-100 \%$, and $86.8-90.2 \%$ identity with other isolates reported in the GenBank database for FBV1 and FMV, respectively. While the FFkaV strains have shown 94.2\% identical between the two Palestinian strains, and $88.3-90.09 \%$ identity with isolates reported in the GenBank database, on the other hand, the two FLMaV-2 strains were $92.2 \%$ identical and showed $86.8-94.4 \%$ with other strains reported in the GenBank database.

The phylogenetic trees constructed based on the se-
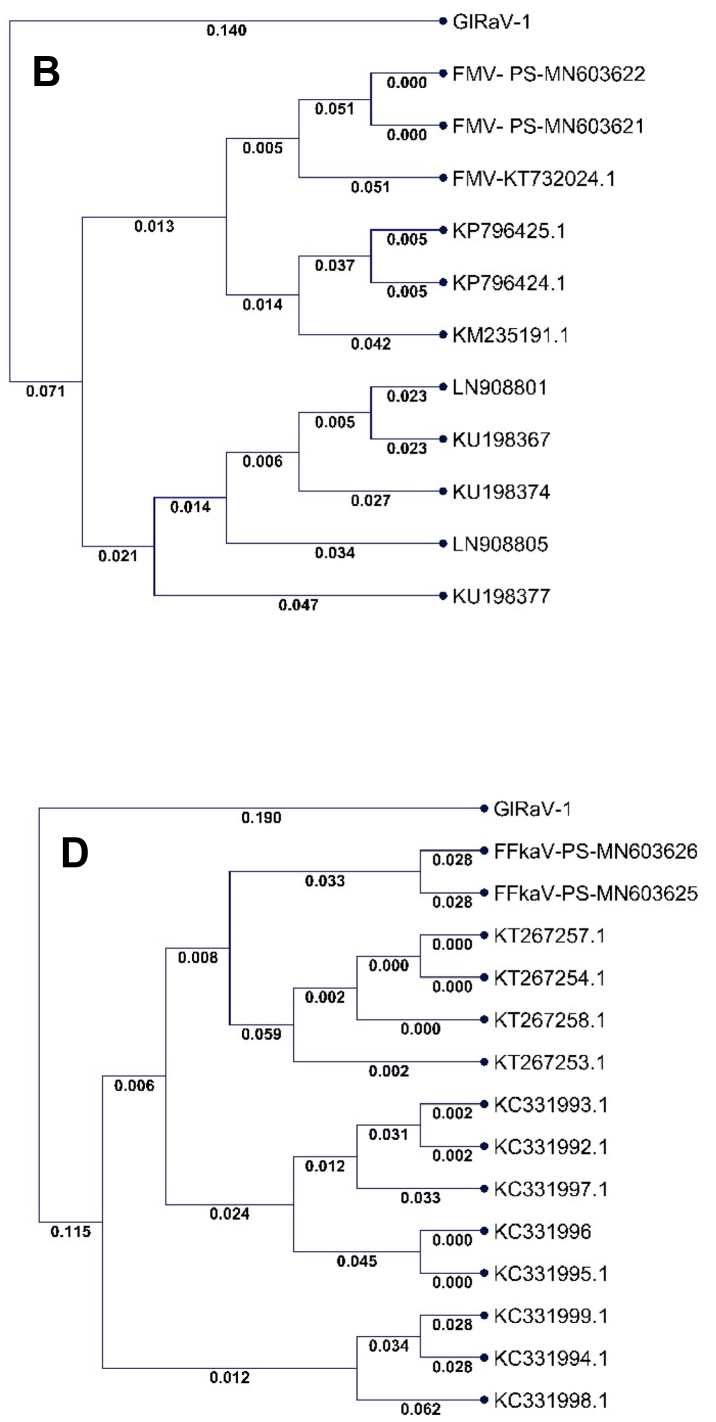

Fig. 5. Phylogenetic trees generated from the alignment of nucleotide sequences for partial genes of Fig badnavirus-1 (FBV-1) (A), Fig mosaic emaravirus (FMV) (B), Fig leaf mottle-associated virus 2 (FLMaV-2) (C), Fig fleck-associated virus (FFkaV) (D), together with homolog sequences reported in the GenBank (Supplementary Table 1), using the neighbor-joining algorithm, p-distance method and bootstrap consisting of 1,000 pseudoreplicates. Nucleotide sequences of Grapevine leafroll-associated virus 1 (GLRaV-1, accession no. KU674797) was used as outgroup species to root the trees. Branch lengths represent bootstrap values. The bar represents 0.08 changes per site. 
quences attained in this study and those retrieved from the GenBank for each virus showed in the case of FBV1 that the two Palestinian isolates are close to each other and grouped in one clade with the Iranian isolates (Fig. 5A). Also, the two FMV Palestinian isolates grouped in the same clade with that from Lorestan province in Iran (Fig. 5B). However, two main clusters were generated in the FLMaV-2 phylogenetic tree, and the Palestinian isolates were grouped in different sub-clusters, one isolate was with the same clade of FLMaV-2 isolate from Bosnia, while the other isolate was on a single clade grouped in a sub-cluster of isolates from different regions (Syria, Albania, Algeria, and Lebanon) (Fig. 5C). In the case of the FFkaV phylogenetic tree, three main clusters were generated, and the Palestinian isolates were grouped in one clade and were in the same bunch of the Turkish strains (Fig. 5D).

\section{Discussion}

In our study, the FMD symptoms were noted on leaves in all surveyed cultivars in Palestine, but with diverse symptoms types and severity expression.

Among the eight viruses screened that can be linked with FMD, our results established the occurrence of four viruses: FMV, FBV-1, FLMaV-2, and FFkaV. The results support those reported by Alkowni et al. (2015), who documented the presence of FBV-1, FMV, FLMaV-2, and FLV-1 in fig leaf samples, during a preliminary survey conducted on a limited number of samples (25 trees) in one small locality in Nablus District in the NWB. In the comparison of these findings with our results, all the tested trees and seedlings (91) from all cultivars, were infected by at least two viruses, of which FMV and FBV-1 were the most predominant viruses with an incidence rate of $100 \%$ in all tested plants, followed by FLMaV-2 (54.5\%) and FF$\mathrm{kaV}(20.8 \%)$ (Table 3). Therefore, we can conclude that so far in the West Bank of Palestine, the following fig viruses were detected: FMV, FBV-1, FLMaV-2, FFkaV, and FLV1 among eight viruses examined in both studies.

The high levels of FMV and FBV-1 infections detected in Palestine are following their widespread incidence in many countries including Bosnia \& Herzegovina, Montenegro, China, Italy, Turkey, Iran, Algeria, and Lebanon (Delić et al., 2017; Laney et al., 2012; Latinović et al., 2019; Mijit et al., 2017; Minafra et al., 2012); and in general, the levels of virus infections obtained here were higher than those earlier reported from other Mediterranean countries (Table 1 and references therein). The high infection rates can be attributed to the clonal propagation of common fig trees (by hardwood cuttings), and the existence of suit- able virus vectors (Aceria ficus, mealybugs, and aphids) (Yahyaoui et al., 2017), both factors promote the spread of viral agents in nature.

FBV-1, the only DNA virus, has shown to be one of the most prevalent viruses, being found in all samples from all trees and newly propagated seedlings of different origins and cultivars tested (at a 100\% detection rate). Tzanetakis et al. (2010) were the first to designate FBV-1 as a new badnavirus affecting fig. Laney et al. (2012) provided evidence on the integration of the virus into the fig genome; the study has also revealed the wide virus distribution in fig in a large number of trees of different origins in the United States (98\% incidence rate). A comparable state was observed in New Zealand, and Turkey, where Minafra et al. (2012), and Delić et al. (2017) detected the virus in 100\% of the samples analyzed. A lower \% (96.4) of infection by FBV-1 was reported by Latinović et al. (2019) in Montenegro; the virus was prevalent among the investigated fig plants. The high incidence of this virus in the newly propagated seedlings from different cultivars may be an indication that FBV-1 integrates into the genome of figs in a cultivar nondiscriminatory manner.

Regarding the relation of FBV-1 and FMD symptom induction, there is mounting evidence that the virus is asymptomatic in single infections, at best in some cultivars (Laney et al., 2012). However, assuming a large number of cultivars existing, this may not be accurate for others. There is also an apparent possibility that fig infection with FBV-1 modifies the internal physiology of the plant, as has been demonstrated with FLMaV-1 (Fayez and Mahmoud, 2011). Badnaviruses are also recognized to play a significant part in viral disease combinations (Jones et al., 2002), and that their participation in mixed infections causes severe symptoms in crops propagated by rooted cuttings (Tzanetakis et al., 2007; Wang and Valkonen, 2008). Latinović et al. (2019) suggested that singly FBV-1 infected plants show mild mosaic symptoms, which cannot be established in our study because the virus exists continuously in mixed infections.

Given the association of FBV-1 with severe FMD and its potential substantial role in virus combinations, it may present a new, non-documented problem for the fig industry, not only in Palestine but also in other parts of the world. The significance of such association by FBV-1 calls for further study to understand its epidemiology and population structure and set the ground for virus control management in the nursery propagation stage.

FMV, in this study, always existed in mixed infections in fig plants, both in symptomatic (with diverse symptoms) and in symptomless trees of several cultivars. This is in 
agreement with Mijit et al. (2017), who also found FMV in mixed infections, in about $80 \%$ of symptomatic samples and nearly $50 \%$ of symptomless samples they collected from China. These findings support the notion that FMD is more intricate than was assumed earlier, and symptoms might not be induced by FMV, even in mixed virus infections, for unknown reasons (Elbeaino et al., 2009a; Walia et al., 2009).

The pathogenicity of other viruses (FLMaV-2 and FF$\mathrm{kaV}$ ) found in plants with FMD is still uncertain. The present study has demonstrated that FLMaV-2, occurs in symptomatic plants with high incidence $(54.5 \%)$ and the majority $(13 / 17,76.5 \%)$ of the tested cultivars. Our results, however, are in agreement with those of other researchers who also found FLMaV-2 to occur in symptomatic fig trees, indicating its association with FMD, in several Mediterranean countries including Syria, Lebanon, Tunisia, Algeria, Italy, Albania, with different extent of infections (Elbeaino et al., 2006, 2007, 2010; Nahdi et al., 2006). The high incidence of FLMaV-2 could be due to high populations on fig plants of an insect likely to be a typical vector, such as Planococcus ficus (vine mealybug), and the fig wax scale insect (Ceroplastes rusci) (Yahyaoui et al., 2017; Yorganci, 2016).

Elbeaino et al. (2011a) were the first to designate FF$\mathrm{kaV}$ as a new Maculavirus of the family Tymoviridae. Its genome comprises a positive sense, single-stranded RNA encoding for replication, coat, and movement proteins. It mostly exists in mixed infections, and attempts to transmit the virus mechanically to various test plants have been unsuccessful (Ale-Agha and Rakhshandehroo, 2014). In our study, FFkaV was always present in combination with other viruses, and it was the virus with the least incidence (20.8\%). This is the first report on the presence of FFkaV in figs in Palestine. Although based on our results, it was not possible to establish a cause-effect relationship, between this virus and specific symptomatology shown by the fig trees (Elbeaino et al., 2011a), the virus appears to be deeply rooted in its host, as it occurs in all studied provinces of Palestine with incidence rates ranging from 11\% (Southern West Bank) to 35.5\% (Northern West Bank). In our study, since all the viruses infecting FMD plants were always in combination with at least two viruses, singly virus-induced symptoms can neither be established nor substantiated.

The present study has investigated the possible association between symptom types and the viruses detected in the 17 Palestinian fig cultivars. In view of the results of various symptom types expressed in the studied FMD plants of different cultivars, it can be concluded that no clear-cut association could be established between the expressed symptom types and specific virus combinations observed in this study (Elbeaino et al., 2011a).

On the bases of the processed binary images of the \%LAWS, there were diverse responses in symptom expression (i.e., disease severity) in the FM trees with various virus combinations: of the 17 fig cultivars nine showed relatively high symptom expression (\% LAWS 12-16), seven showed medium symptom expression (6-10\%); and one cultivar showed the lowest symptom expression (2\%) with a virus combination of FMV, FBV-1, and FLMaV-2 (Table 4$)$. In general, very weak or no association $\left(r^{2}=0.05\right)$ was detected between FMD symptoms severity in the 17 Palestinian fig cultivars with the various viruses' combinations observed in terms $\%$ of positive signals. These results show that FMD symptoms severity might be closely associated with cultivar type, rather than the number of viruses infecting the plant as indicated by Elbeshehy and Elbeaino (2011); and Martin et al. (2013).

In conclusion, the leaves of all surveyed fig cultivars in Palestine displayed FMD with various severities. After RTPCR and sequencing, among the detected viruses, evidence was attained for the occurrence of four viruses: FMV, FBV-1, FLMaV-2, and FFkaV. FMV and FBV-1 were the most prevalent viruses, being found in all the tested fig cultivars FLMaV-2 was the second most pervasive; it was present in all surveyed cultivars, excluding Khortmani, Mwazi, Ghzali, Sfari. The incidence of FFkaV was low in the tested fig plants. All of the viruses were present in combination with other viruses. Concerning the processed binary images of the \%LAWS, there were diverse responses in symptom expression in the FM trees of the 17 fig cultivars. These results support the notion that FMD symptoms severity might be closely associated with cultivar type, rather than the number of viruses infecting the plant.

\section{Acknowledgments}

This research was supported by a grant from the Biodiversity and Environmental Research Center, BERC.

\section{Electronic Supplementary Material}

Supplementary materials are available at The Plant Pathology Journal website (http://www.ppjonline.org/).

\section{References}

Aldhebiani, A. Y., Elbeshehy E. K. F., Baeshen, A. A. and Elbeaino, T. 2015. Four viruses infecting figs in Western Saudi Arabia. Phytopathol. Mediterr. 54:497-503. 
Ale-Agha, G. N. and Rakhshandehroo, F. 2014. Detection and molecular variability of fig fleck-associated virus and fig cryptic virus in Iran. J. Phytopathol. 162:417-425.

Ali-Shtayeh, M. S., Jabi, F. F. and Hamad, A. K. 1991. The fig tree. Rural Research Centre, An-Najah National University, Nablus, Palestine. 137 pp. (in Arabic).

Ali-Shtayeh, M. S., Jamous, R. M., Abu Zaitoun, S. Y., Mallah, O. B. and Mubaslat, A. K. 2014. Genetic diversity of the Palestinian fig (Ficus carica L.) collection by pomological traits and RAPD markers. Am. J. Plant Sci. 5:1139-1155.

Alkowni, R., Chiumenti, M., Minafra, A. and Martelli, G. P. 2015. A survey for fig-infecting viruses in Palestine. J. Plant Pathol. 2:383-386.

Caglar, B. K., Fidan, H., Guldur, M. E. and Elbeaino, T. 2011. The prevalence of three viruses infecting fig in Southern Turkey. J. Phytopathol. 159:181-183.

Delić, D., Perović, T., Hrnčić, S., Lolić, B., Durić, G. and Elbeaino, T. 2017. Detection and phylogenetic analyses of figinfecting viruses in Bosnia and Herzegovina and Montenegro. Phytopathol. Mediterr. 56:470-478.

El Air, M., Mahfoudhi, N., Digiaro, M., Dhouibi, M. H. and Elbeaino, T. 2015. Incidence and distribution of viruses in Tunisian fig orchards. J. Plant Pathol. 97:327-331.

Elbeaino, T., Abou Kubaa, R., Ismaeil, F., Mando, J. and Digiaro, M. 2012a. Viruses and hop stunt viroid of fig trees in Syria. $J$. Plant Pathol. 94:687-691.

Elbeaino, T., Choueiri, E., Hobeika, C. and Digiaro, M. 2007. Presence of Fig leaf mottle-associated virus 1 and 2 in Lebanese fig orchards. J. Plant Pathol. 89:409-411.

Elbeaino, T., Digiaro, M., Alabdullah, A., De Stradis, A., Minafra, A., Mielke, N., Castellano, M. A. and Martelli, G. P. 2009a. A multipartite single-stranded negative-sense RNA virus is the putative agent of fig mosaic disease. J. Gen. Virol. 90:12811288.

Elbeaino, T., Digiaro, M., De Stradis, A. and Martelli, G. P. 2006. Partial characterisation of a closterovirus associated with a chlorotic mottling of fig. J. Plant Pathol. 88:187-192.

Elbeaino, T., Digiaro, M., Heinoun, K., De Stradis, A. and Martelli, G. P. 2010. Fig mild mottle-associated virus, a novel closterovirus infecting fig. J. Plant Pathol. 92:165-172.

Elbeaino, T., Digiaro, M. and Martelli, G. P. 2011a. Complete sequence of Fig fleck-associated virus, a novel member of the family Tymoviridae. Virus Res. 161:198-202.

Elbeaino, T., Kiyi, H., Boutarfa, R., Minafra, A., Martelli, G. P. and Digiaro, M. 2014. Phylogenetic and recombination analysis of the homing protein domain of grapevine fanleaf virus (GFLV) isolates associated with 'yellow mosaic' and 'infectious malformation' syndromes in grapevine. Arch. Virol. 159:2757-2764.

Elbeaino, T., Kubaa, R. A., Digiaro, M., Minafra, A. and Martelli, G. P. 2011b. The complete nucleotide sequence and genome organization of Fig cryptic virus, a novel bipartite dsRNA virus infecting fig, widely distributed in the Mediterranean basin. Virus Genes 42:415-421.
Elbeaino, T., Kubaa, R. A., Ismaeil, F., Mando, J. and Digiaro, M. 2012b. Viruses and Hop stunt viroid of fig trees in Syria. J. Plant Pathol. 94:687-691.

Elbeaino, T., Mortada, C., Digiaro, M. and Choueiri, E. 2012c. Survey on fig viruses in Lebanon. Acta Hortic. 940:665-668.

Elbeaino, T., Nahdi, S., Digiaro, M., Alabdullah, A. and Martelli, G. P. 2009b. Detection of FLMaV-1 and FLMaV-2 in the Mediterranean region and study on sequence variation of the hsp70 gene. J. Plant Pathol. 91:425-431.

Elbeshehy, E. K. F. and Elbeaino, T. 2011. Viruses infecting figs in Egypt. Phytopathol. Mediterr. 50:327-332.

Fayez, K. A. and Mahmoud, S. Y. 2011. Detection and partial characterization of a putative closterovirus affecting Ficus carica: molecular, ultrastructural and physiological aspects of infected leaves. Acta Physiol. Plant 33:2187-2198.

Flock, R. A. and Wallace, J. M. 1955. Transmission of fig mosaic by the eriophyid mite Aceria ficus. Phytopathology 45:52-54.

Foissac, X., Svanella-Dumas, L., Dulucq, M. J., Candresse, T. and Gentit, P. 2001. Polyvalent detection of fruit tree tricho, capillo and foveaviruses by nested RT-PCR using degenerated and inosine containing primers (PDO RT-PCR). Acta Hortic. 550:37-44.

Food and Agriculture Organization of the United Nations. 2009. The FAO Statistical Database-Agriculture. URL http://faostat3.fao.org/faostat-gateway/go/to/download/Q/QC/E [9 January 2020].

Gattoni, G., Minafra, A., Castellano, M. A., De Stradis, A., Boscia, D., Elbeaino, T., Digiaro, M. and Martelli, G. P. 2009. Some properties of fig latent virus 1 , a new member of the family Flexiviridae. J. Plant Pathol. 91:555-564.

Goor, A. 1955. The fig. Agricultural Publication Section, Department of Horticulture, Department of Agriculture Education, Tel Aviv, Israel. 70 pp. (in Hebrew).

Jones, A. T., McGavin, W. J., Geering, A. D. W. and Lockheart, B. E. L. 2002. Identification of Rubus yellow net virus as a distinct badnavirus and its detection by PCR in Rubus species and in aphids. Ann. Appl. Biol. 141:1-10.

Laney, A. G., Hassan, M. and Tzanetakis, I. E. 2012. An integrated badnavirus is prevalent in fig germplasm. Phytopathology 102:1182-1189.

Latinović, J., Radišek, S., Bajčeta, M., Jakše, J. and Latinović, N. 2019. Viruses associated with fig mosaic disease in different fig varieties in Montenegro. Plant Pathol. J. 35:32-40.

Martin, R. R., MacFarlane, S., Sabanadzovic, S., Quito, D., Poudel, B. and Tzanetakis, I. E. 2013. Viruses and virus diseases of Rubus. Plant Dis. 97:168-182.

Mijit, M., He, Z., Hong, J., Lu, M.-G., Li, S.-F. and Zhang, Z.-X. 2017. Analysis of fig tree virus type and distribution in China. J. Integr. Agric. 16:1417-1421.

Minafra, A., Chiumenti, M., Elbeaino, T., Digiaro, M., Bottalico, G., Pantaleo, V. and Martelli, G. P. 2012. Occurrence of fig badnavirus 1 in fig trees from different countries and in symptomless seedlings. J. Plant Pathol. 94(4 Suppl):S4.105.

Nahdi, S., Elbeaino, T., Digiaro, M. and Martelli, G. P. 2006. 
First record of Fig leaf mottle-associated virus 1 in Tunisia. $J$. Plant Pathol. 88(3 Suppl):S70.

Norozian, E., Rakhshandehroo, F. and Shams-Bakhsh, M. 2014. Presence of fig leaf mottle-associated virus 3 in an Iranian fig orchard. J. Plant Pathol. 96(4 Suppl):S4.131.

Pethybridge, S. J. and Nelson, S. C. 2015. Leaf doctor: a new portable application for quantifying plant disease severity. Plant Dis. 99:1310-1316.

Sambrook, J., Fritsch, E. F. and Maniatis, T. 1989. Molecular cloning: a laboratory manual. 2nd ed. Cold spring harbor laboratory, Cold Spring Harbor, NY, USA. 1626 pp.

Shahmirzaie, M., Rakhshandehroo, F., Zamanizadeh, H. R. and Elbeaino, T. 2012. Current status of fig mosaic disease in Iran. J. Phytopathol. 160:324-330.

Susaimuthu, J., Tzanetakis, I. E., Gergerich, R. C. and Martin, R. R. 2008. A member of a new genus in the Potyviridae infects Rubus. Virus Res. 131:145-151.

Tzanetakis, I. E., Halgren, A., Mosier, N. and Martin, R. R. 2007. Identification and characterization of Raspberry mottle virus, a novel member of the Closteroviridae. Virus Res. 127:26-33.

Tzanetakis, I. E., Laney, A. G., Keller, K. E. and Martin, R. R. 2010. New viruses found in fig exhibiting mosaic symptoms.
In: 21st International Conference on Virus and other Graft Transmissible Deseases of Fruit Crops, eds. by W. Jelkmann, G. Krczal and F. Feldmann, pp. 79-82. Julius Kühn-Institut, Berlin, Germany.

Walia, J. J., Salem, N. M. and Falk, B. W. 2009. Partial sequence and survey analysis identify a multipartite, negative-sense RNA virus associated with fig mosaic. Plant Dis. 93:4-10.

Wang, Q. C. and Valkonen, J. P. T. 2008. Elimination of two viruses which interact synergistically from sweetpotato by shoot tip culture and cryotherapy. J. Virol. Methods 154:135145.

Yahyaoui, E., Frasheri, D., Germanà, M. A., Burruano, S., D’Onghia, A. M. and Elbeaino, T. 2017. Viruses infecting different Mediterranean genotypes of Ficus carica and their distribution in different plant organs. Acta Hortic. 1173:273278.

Yorganci, S. 2016. Determination of some vectors for fig mosaic disease agents transmission. MSc thesis. Adnan Menderes Üniversitesi, Aydın, Turkey (in Turkish).

Zeven, A. C. 1998. Landraces: a review of definitions and classifications. Euphytica 104:127-139. 